\title{
ON THE USE OF DEEP, SHALLOW OR FLAT SHELL FINITE ELEMENTS FOR THE ANALYSIS OF THIN SHELL STRUCTURES
}

\author{
Sergio IDELSOHN \\ Aerospace Laboratory of the University of Liège, Rue Ernest Solvay, 21, B-4000 Liège, Belgium \\ Received 30 April 1980 \\ Revised manuscript received 22 August 1980
}

\begin{abstract}
This paper is confined to the study of thin shells. The aim is to summarize the different theories used and to examine the assumptions upon which each of them is based. The intention is to show when it is more suitable to use a particular approximation and to indicate the errors it introduces. Beginning with the general deep shell theory, some simplifications are introduced to obtain the shallow shell theories. The special implications of this theory for the finite element method are also examined. Finally the particular case of flat elements is discussed.
\end{abstract}

\section{Deep shell theory}

A shell, by definition, is a body limited by two surfaces separated from each other by a distance $h$, which is small in relation to its other dimensions.

This geometrical characteristic allows the three dimensional elasticity theory to be reduced to a two-dimensional theory.

If the correct hypothesis describing the transversal behaviour of the shell is found, all the calculations may be carried out in terms of the middle surface points.

This middle surface is defined by a vector $r$, the components of which are the curvilinear coordinates $x^{1}, x^{2}$.

The two base vectors $a_{\alpha}$ tangential to the middle surface are obtained as the derivatives of $r$ with respect to each curvilinear coordinate. The metric surface tensor $a_{\alpha \beta}$ and curvature tensor of the middle surface $b_{\alpha \beta}$ are obtained as indicated in fig. 1. In this paper Greek indexes range from 1 to 2 . A single stroke stands for covariant differentiation with respect to the surface metric and a comma denotes partial differentiation with respect to the curvilinear coordinate $x^{\alpha}$.

Excepting the case of thick shells, all shell theories are based on neglecting the effect of normal stresses and assuming that the displacements or the stresses parallel to the middle surface vary linearly across the thickness.

With these assumptions all the displacement fields may be completely defined by the middle surface displacements and the middle surface rotations [1].

The suplementary hypothesis made for thin shell is to identify the rotation components $\omega_{\alpha}$ with the normal rotation of the middle surface $\phi_{\alpha}$.

For this particular shell, it has been shown [2], that the strain energy can be explained as the sum of two terms: the stretching energy and the bending energy. The assumption involved in 


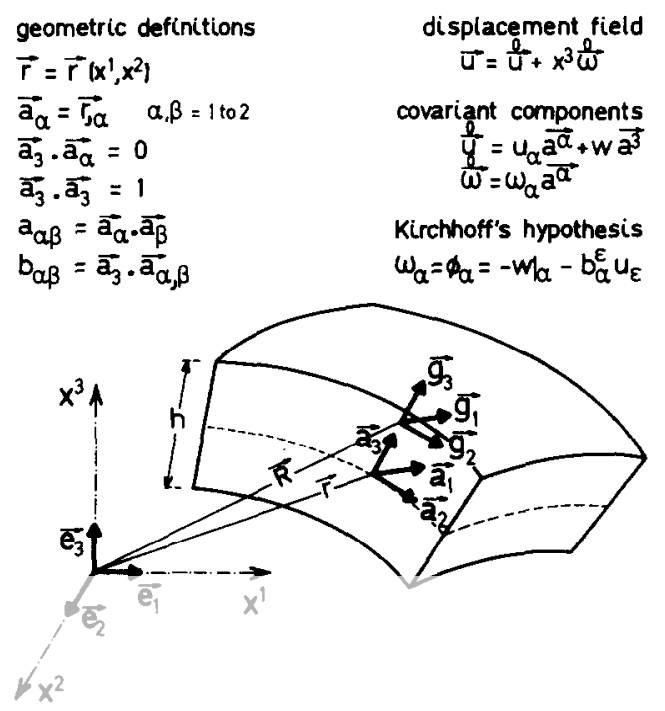

Fig. 1. Shell element.

neglecting the connecting terms is consistent with Kirchhoff's hypothesis. In linear elasticity,

the middle surface strain tensor $\gamma_{\alpha \beta}$ and the curvature change tensor $\rho_{\alpha \beta}$ are:
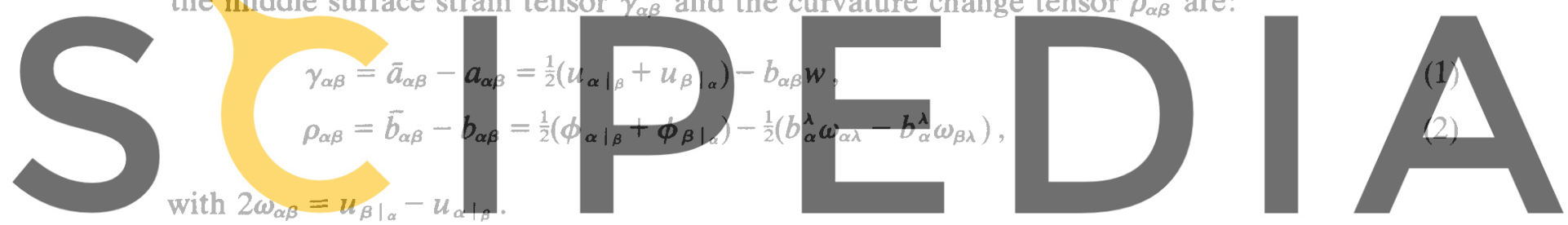

In these equations the superimposed line stands for a tensor after deformation. All these

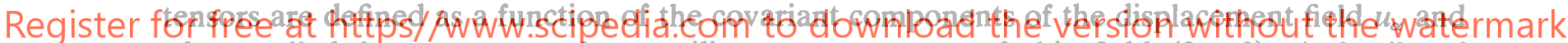
of $w$, called from now on the curvilinear components of this field (fig. 2). A family of conforming finite elements based on this formulation has been developed by the author [3].

Each element of the family is obtained by subdividing the triangular or quadrangular element into three or four regions, in each of which a complete cubic polynomial field is assumed for the transversal displacement component and a complete $n$-degree polynomial for

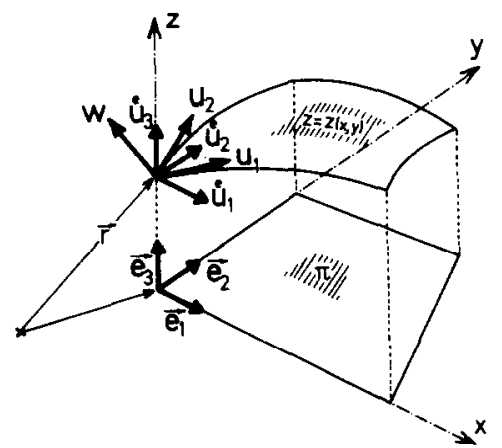

Fig. 2. Covariant and cartesian components of the displacement field. 
the tangential displacement field. The strain energy is obtained as a function of the complete strain tensor $\gamma_{\alpha \beta}$ and the curvature change tensor $\rho_{\alpha \beta}$ of eqs. (1) and (2). Numerical integration is carried out to achieve the stiffness matrix. After assembling the stretching and the bending part, each element contains three displacement components and two rotational components at each corner node. Along the interfaces, a tangential rotation is always present, while there can be zero, one or two tangential displacements depending on the degree of the polynomial approximation. This general deep shell element is named GDSi, where $i$ is the degree of the tangential displacement field (fig. 3a).

Another way of stating the shell theory is to calculate the strain tensor as a function of the cartesian displacement components (which are parallel to the cartesian base vector $e$ ) denoted here $\stackrel{e}{u}_{1}, \stackrel{e}{u}_{2}$ and $\stackrel{e}{u_{3}}$ (fig. 2). The advantage of this formulation is a proper representation of the rigid-body motions when a finite element approach is used. A deep shell element based on this theory has been developed in [4].

\section{The shallow shell theory in curvilinear components}

In some cases it is possible to define a plane nearly parallel to the middle surface of the shell. If this plane is used as a reference plane and two of the cartesian components of the vector $r$ are chosen to be parallel to the plane and the third to be perpendicular, the shell

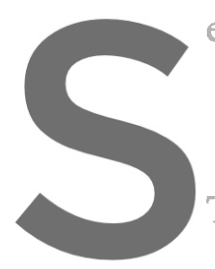

equation becomes
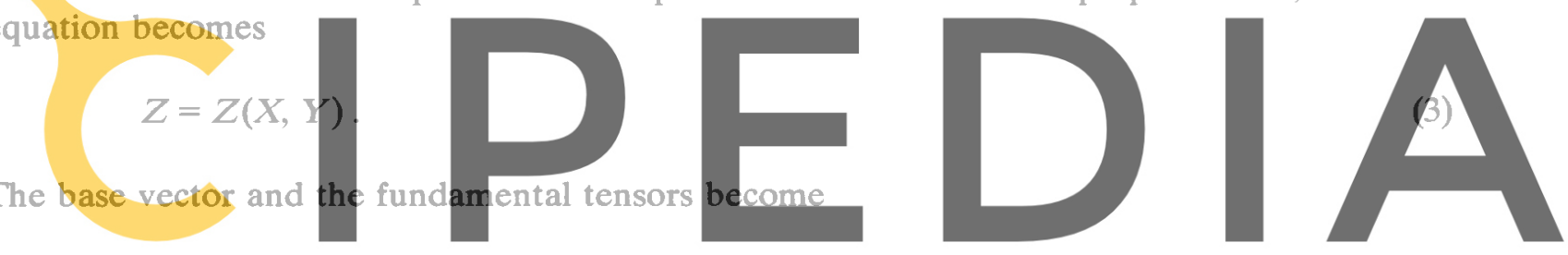

$$
a_{\alpha}=r_{, \alpha}=e_{\alpha}+Z_{, \alpha} e_{3}
$$

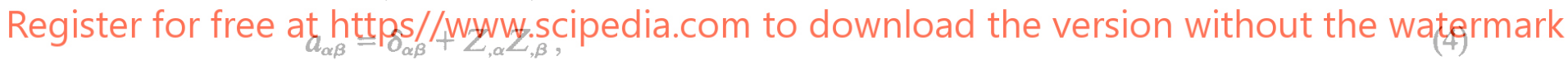

$$
\begin{aligned}
& b_{\alpha \beta}=Z_{, \alpha \beta} / \sqrt{a} \text { with } a=1 / Z_{, \alpha}^{2}+Z_{, \beta}^{2}, \\
& \boldsymbol{b}_{\boldsymbol{\alpha}}^{\boldsymbol{\beta}}=\left(\boldsymbol{Z}_{, \boldsymbol{\alpha} \boldsymbol{\beta}}+Z_{, \boldsymbol{\gamma} \boldsymbol{\alpha}} Z_{, \boldsymbol{\alpha}} Z_{, \boldsymbol{\beta}}(\mathbf{- 1})^{\boldsymbol{\gamma} \boldsymbol{\beta}}\right) / \boldsymbol{a}^{\mathbf{3 / 2}} .
\end{aligned}
$$

The strain tensor and curvature change tensor in curvilinear components can be obtained from (1), (2) and (4). Developing the covariant differentiation, they can be written as the sum of two parts:

$$
\begin{gathered}
\gamma_{\alpha \beta}=\stackrel{s}{\gamma_{\alpha \beta}}+\stackrel{D}{\gamma_{\alpha \beta},}, \\
\rho_{\alpha \beta}=\stackrel{s}{\rho_{\alpha \beta}}+\stackrel{D}{\rho_{\alpha \beta},},
\end{gathered}
$$

with

$$
\stackrel{s}{\gamma \beta}_{\alpha \beta}=\frac{1}{2}\left(u_{\alpha, \beta}+u_{\beta, \alpha}\right)-b_{\alpha \beta} w,
$$




$$
\begin{aligned}
& \stackrel{D}{\gamma_{\alpha \beta}}=-\frac{1}{2}\left[u_{\lambda} a^{\varepsilon \lambda}\left(a_{\varepsilon \alpha, \beta}-a_{\alpha \beta, \varepsilon}+a_{\beta \varepsilon, \alpha}\right)\right], \\
& \stackrel{s}{\rho_{\alpha \beta}}=-w_{, \alpha \beta}, \\
& \stackrel{D}{\rho}_{\alpha \beta}=\frac{1}{2}\left[-b_{\alpha}^{\lambda} u_{\lambda_{, \beta}}-b_{\alpha_{\beta}}^{\lambda} u_{\lambda}-b_{\beta}^{\lambda} u_{\lambda_{, \alpha}}-b_{\beta, \alpha}^{\lambda} u_{\lambda}\right. \\
& \left.-\phi_{\lambda} a^{\varepsilon \lambda}\left(a_{\varepsilon \alpha, \beta}-a_{\alpha \beta, \varepsilon}+a_{\beta \varepsilon, \alpha}\right)-b_{\beta}^{\lambda}\left(u_{\lambda, \beta}-u_{\beta, \lambda}\right) b_{\alpha}^{\lambda}\left(u_{\lambda, \alpha}-u_{\alpha, \lambda}\right)\right] .
\end{aligned}
$$

The shallow shell theory assumes that

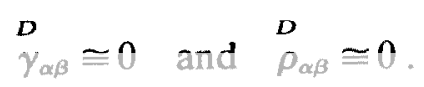

In fact, there are two simplifying hypotheses in the shallow shell theory. The first is a geometric assumption:

$$
Z_{, \alpha} \ll 1,
$$

i.e., the surface slopes with respect to the reference plane are small

The second is a displ

ments are small in relation to the transversal
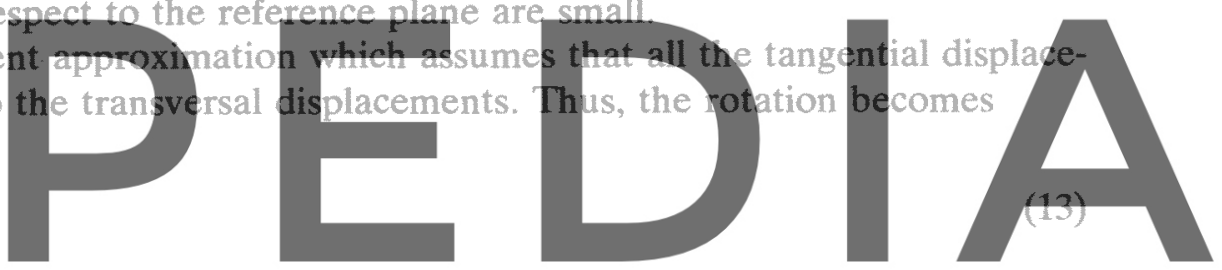

and the strain tensors read

Register for free at https//www.scipedia.com to download the version without the watermark

$$
\begin{aligned}
& \gamma_{\alpha \beta}=\frac{1}{2}\left(u_{\alpha, \beta}+u_{\beta, \alpha}\right)-Z_{, \alpha \beta} W \\
& \boldsymbol{\rho}_{\boldsymbol{\alpha} \boldsymbol{\beta}}=-\boldsymbol{w}_{. \boldsymbol{\alpha \beta}} .
\end{aligned}
$$

\section{The shallow shell theory in cartesian components}

On the other hand, using the cartesian displacement components and the assumptions (3) and (4), the strain tensors become [5]:

$$
\begin{aligned}
& \gamma_{\alpha \beta}=\frac{1}{2}\left(\dot{u}_{\alpha, \beta}+\stackrel{e}{u}_{\beta, \alpha}+Z_{, \alpha} \dot{e}_{3, \beta}+Z_{, \beta} \dot{e}_{3, \alpha}\right), \\
& \rho_{\alpha \beta}=\stackrel{M}{\rho_{\alpha \beta}}+\stackrel{N}{\rho_{\alpha \beta}},
\end{aligned}
$$

with 


$$
\begin{aligned}
& \begin{array}{l}
M \\
\rho_{\alpha \beta}
\end{array}=-\stackrel{e}{u_{3, \alpha \beta}} / \sqrt{a} \\
& \stackrel{N}{\rho_{\alpha \beta}}=\left[Z_{, \alpha \beta}\left(Z_{, \nu} Z_{, \lambda} \stackrel{e}{u_{\nu, \lambda}}-Z_{, v} e_{3, \nu}\right) / a-Z_{, \nu} e_{\nu, \alpha \beta}\right] / \sqrt{a} .
\end{aligned}
$$

Here again, the shallow shell assumption is [6]:

$$
\stackrel{M}{\rho_{\alpha \beta}} \simeq 0
$$

and the strain tensors become

$$
\begin{aligned}
& \gamma_{\alpha \beta}=\frac{1}{2}\left(u_{\alpha, \beta}^{e}+\stackrel{e}{u_{\beta, \alpha}}+Z_{, \alpha}^{e} u_{3, \beta}+Z_{, \beta} e_{3, \alpha}^{e}\right), \\
& \rho_{\alpha \beta}=-u_{3, \alpha \beta} .
\end{aligned}
$$

4. Special implications of the shallow shell theory in the finite element method

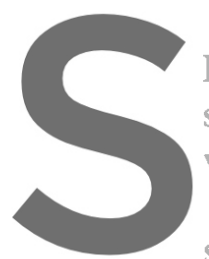

It is important to

First, the geometric ass second assumption is valid if only a part of th

In the finite element shell theory. In this method, the whole structu

can be treated as a shallow shell. However, the displacement hypotheses of shallow shells are

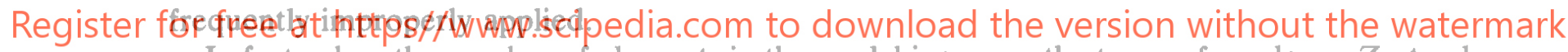

In fact, when the number of elements in the model increases the two surface slopes $Z_{, \alpha}$ tend to zero. However, the second derivatives $Z_{\alpha \beta}$ tend to a constant value proportional to the inverse curvature ratio $\boldsymbol{R}_{\alpha \beta}$. Taking into account eqs. (4), this means that

$a_{\alpha \beta}$ tends to $\delta_{\alpha \beta}$,

$a$ tends to 1 ,

$b_{\alpha \beta}$ and $b_{\alpha}^{\beta}$ tends to $Z_{, \alpha \beta}$, and

$a_{\alpha \beta, \gamma}=\left(Z_{, \alpha \gamma} Z_{, \beta}+Z_{, \alpha} Z_{, \beta \gamma}\right)$ tends to 0 .

\subsection{Curvilinear components}

Inspection of eq. (8) reveals that all the terms of ${ }_{\alpha \beta}^{D}$ are multiplied by $a_{\alpha \beta, \gamma}$ thus they vanish when the number of elements increases. These considerations justify the shallow shell assumption of neglecting those terms. Nevertheless, eq. (10) shows that it is not always possible to

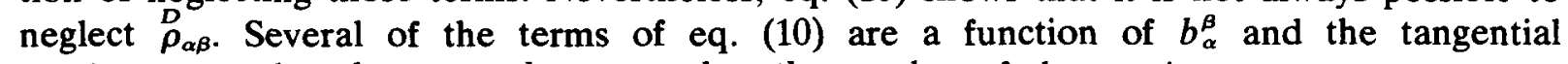
displacements thus do not tend to zero when the number of element increases.

When a deep shell structure is analysed with a shallow shell element idealization, the results 
converge towards a different solution. The difference between the deep shell energy and the shallow shell energy can be evaluated as a function of the neglected terms that do not tend to zero

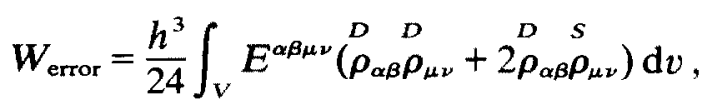

where $h$ is the thickness of the shell and $E^{\alpha \beta \mu \nu}$ the elastic constants in curvilinear coordinates.

However, as it is shown in [7], a deep shell structure can be analysed by shallow shell element in several cases. For instance when the rotations of the shell around the normal

$$
\omega_{\alpha \beta}=\frac{1}{2}\left(\mu_{\left.\beta\right|_{\alpha}}-\mu_{\left.\alpha\right|_{\beta}}\right)
$$

are of the same order as the strain tensor $\gamma_{\alpha \beta}$.

To test this energy gap, a doubly curved family of finite elements has been developed by the author using the shallow shell theory [7]. The characteristics of the elements are the same as for the deep shell elements described above, but the strain energy is a function of the shallow strain tensor equations (14) and (15). Those elements are named GSSi in [7].

A typical shallow shell element based on this theory, was developed by Olson [10] and is shown in fig. 3 b.
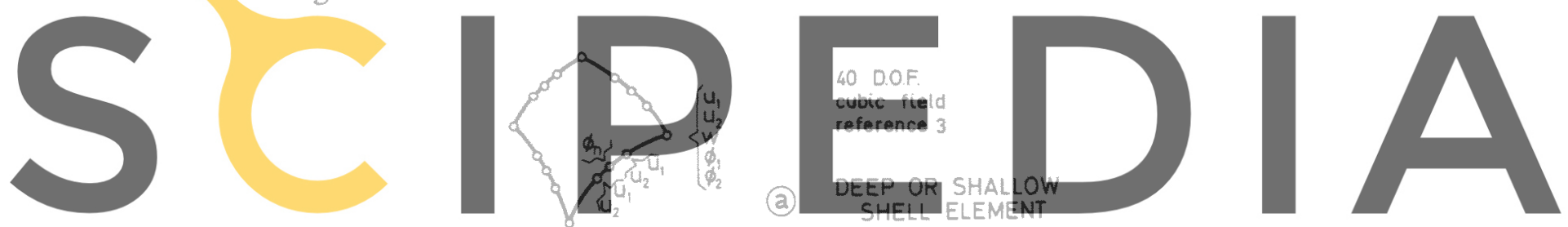

Register for free at https//www.scipedia.com to download the version without the watermark
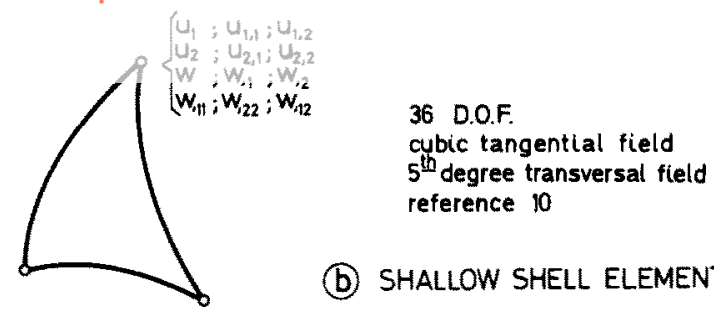

(b) SHALLOW SHELL ELEMENT

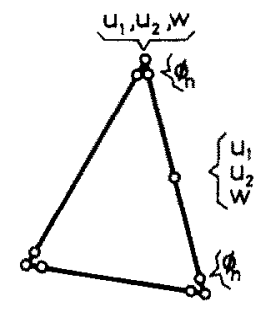

24 D.O.F.

quadratic tangential field

linear bending moments

reference 12

(C) fLAT SHELL ELEMENT

Fig. 3. Some shell finite elements. 


\subsection{Cartesian components}

On the other hand, if the strain tensors are taken as function of the cartesian displacement components, the terms neglected in the shallow shell theory are $\boldsymbol{N}_{\boldsymbol{\alpha} \beta}$. All the terms of (19) are multiplied by $Z_{, \alpha}$ or $Z_{, \beta}$ and therefore tend to zero when the element number increases. In this case the strain energy converges to the same solution as that arrived at by using the deep shell theory.

It is important to emphasize here that although the assumptions of the two shallow shell theories are the same, the terms neglected are not. This is because in the latter, the simplifications are made on the cartesian displacement components instead of the curvilinear displacement components. This difference explains the different behaviours of those two shallow shell theories at the limit.

\section{The flat shell elements}

One of the most important aspects of the finite element analysis of shells are the flat shell elements. They are the simplest to generate and are easy to define geometrically. They can be obtained from eqs. (21) and (22) by neglecting the last two terms of (21).

The equations of the strain tensor and curvature change tensor become the well known stretching and plate bending tensors
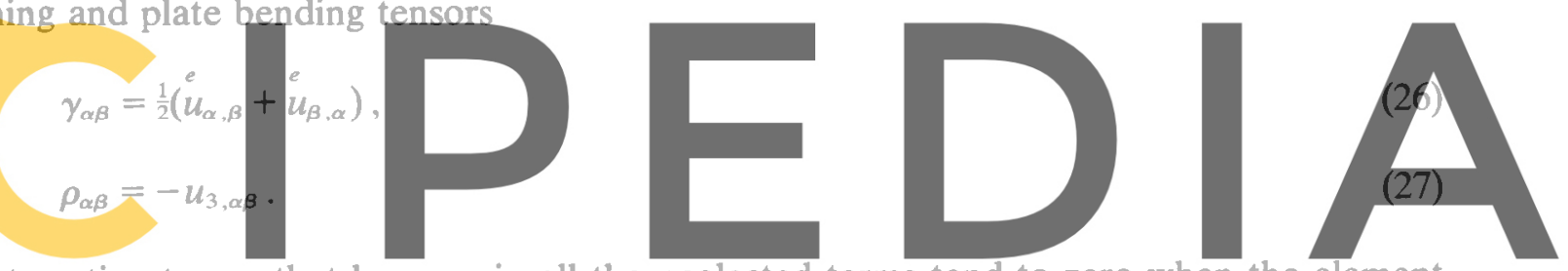

It is interesting to see that here again all the neglected terms tend to zero when the element

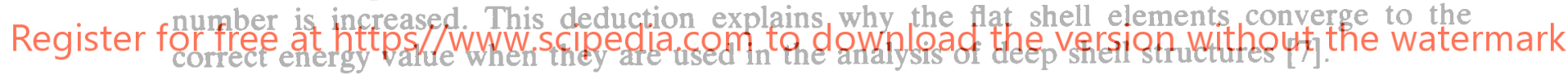

The flat shell approximation cannot be obtained from the curvilinear component shell theory. This is because flat eiements are by deninition functions of the cartesian displacement components.

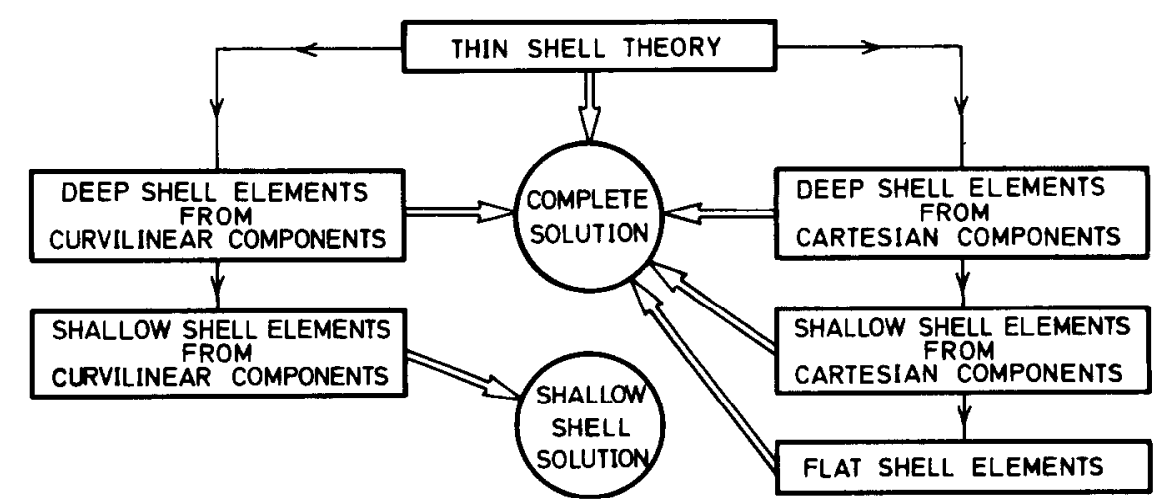

Fig. 4. Flow diagram of the different shell theories. 
Fig. 3c shows a flat shell element developed by the author [12].

The flow diagram of the different theories and their final solution when the element number is increased is shown in fig. 4. Beginning with the general thin shell theory, it is possible to build elements using either, the curvilinear displacement components or the cartesian displacement components. Both models converge to the complete thin shell solution. Shallow shell elements can be obtained as a simplification of both models, but the curvilinear components model does not converge to the complete solution. Finally, it is only possible to obtain the flat elements from a simplification of the cartesian components model and these also converge to the complete thin shell solution.

\section{Numerical results}

The classical case of a cylindrical shell supported at the end by two diaphragms and submitted to its own weight has been analysed here by different elements.

Scordelis and Lo [8] have presented an analytical solution to this problem using the shallow shell theory derived from the curvilinear components.

Fig. 5 shows the shell dimensions and the numerical values used. From symmetry, only a quarter of the shell was needed for the analysis. It has been subdivised into $1 \times 1,2 \times 2,3 \times 3$ and $4 \times 4$ mesh.
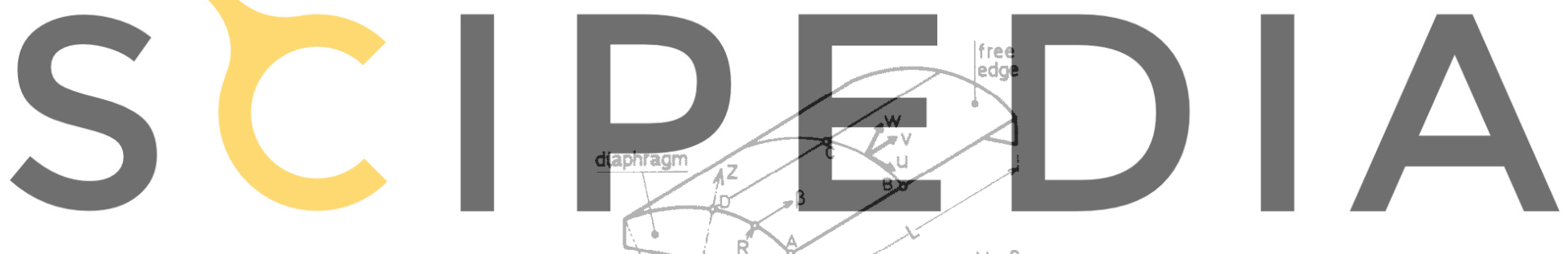

Register for free at https//www.scipediascom to download=ekline version without the watermark

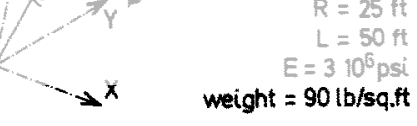

Fig. 5. Cylindrical shell dimensions.

The vertical displacement at the center of the free edge is shown in fig. 6 for several deep, shallow and flat shell elements.

The shallow shell elements compared were the GSS3 with cubic fields developed by the author, in ref. [7], two shallow shell elements developed by Bonnes [9] with cubic fields, a shallow shell element presented by Olson [10] with cubic tangential field and 5th degree for the transversal field, a simple element developed by Strickland [11] with linear tangential fields. All those elements converge to the shallow shell analytical solution of Scordelis and Lo. In particular with the GSS3 element the exact solution is obtained with only 100 d.o.f.

When the complete deep shell theory is used, the solution converges to a result $3.5 \%$ lower, as it can be seen using the GDS3 element.

As it was shown before the solution yielded by flat elements tends to the complete deep shell solution. 


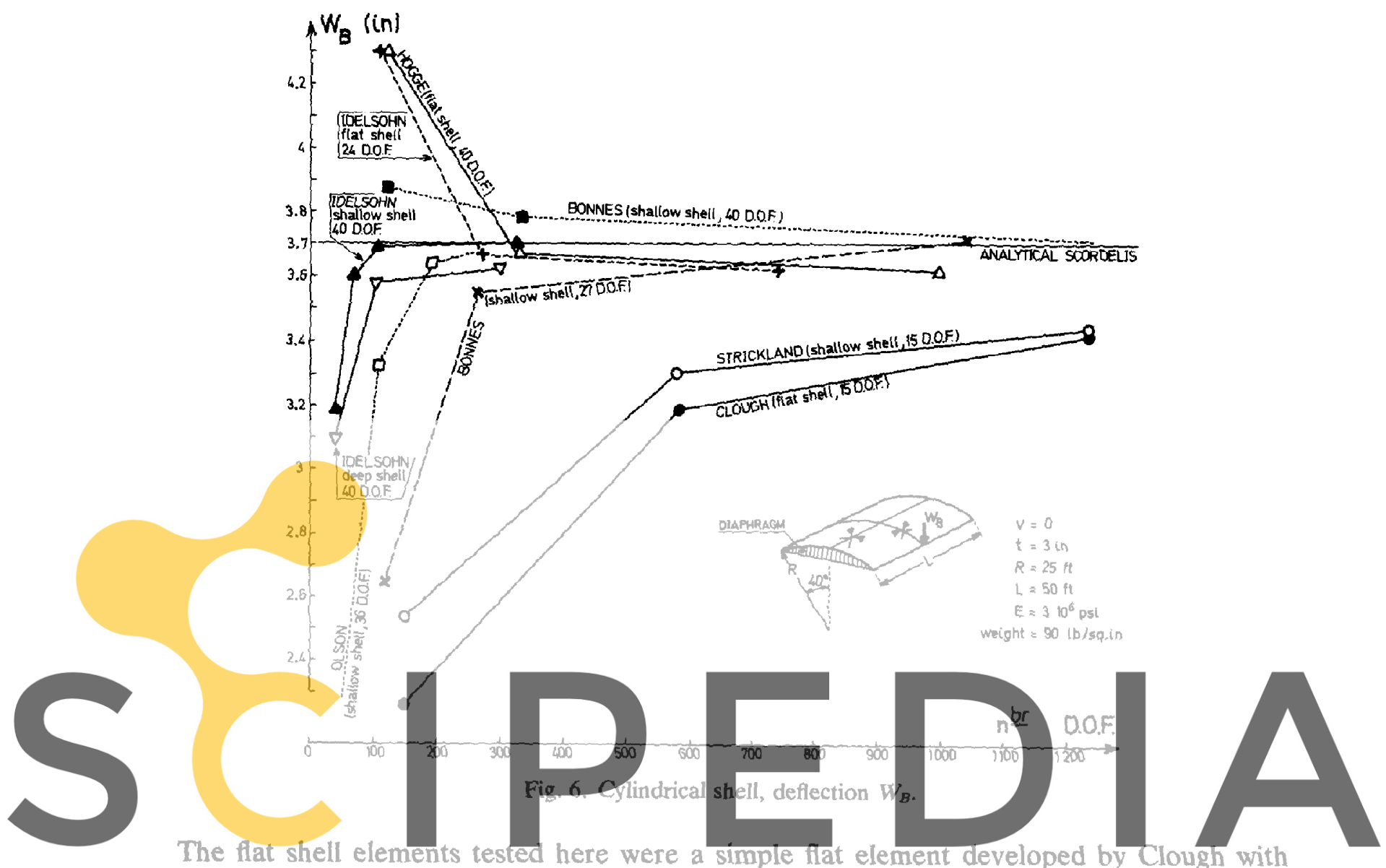
linear tangential displacements and cubic transversal field, a flat shell element with quadratic

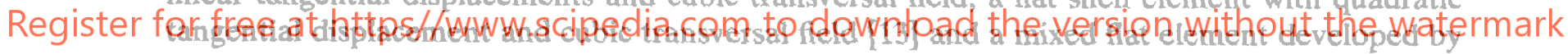
the author with quadratic displacement field for the stretching part and linear moments $M_{x} M_{y}$ and $M_{x y}$ for the bending part [12].

It is important to note that a difference of $3.5 \%$ is obtained between shallow shell elements and deep shell elements although this is a structure that is nearly a shallow shell. For a real deep shell structure, this difference can be much greater.

Many other authors have obtained the deep shell solution with thin shell or degenerate thick shell elements [15-18] but more sophisticated deep shell elements, like SHEBA [14, 19], converge with few elements to the shallow shell results. It should be interesting to confirm this behaviour with other deep shell elements (e.g. [5]) and the new flat shell elements described in [20].

\section{Conclusions}

Shallow thin shell theory is an interesting simplification of the general thin shell theory but significant errors can be found when shallow shell elements in curvilinear components are used for the analysis of deep shell structures. On the other hand, flat shell elements are apparently 
more interesting. They are simple to generate, their geometry is easy to define and the most important conclusion of this paper is that flat shell elements have the property of converging to the correct solution of deep shell theory.

An alternative solution is to build shallow shell elements using the cartesian displacement components (eqs. (21) and (22)). To the author's knowledge, no thin shell elements based on this theory have yet been developed.

\section{References}

[1] B.M. Fraeijs de Veubeke, Dual analysis by finite elements non linear shell theory, USAF Tech. Report AFFDL-TR-72, 93, Wright-Patterson Air Force Base (1971).

[2] W.T. Koiter, A consistant first approximation in the general theory of thin shell, Proc. IUTAM Symposium on the Theory of Thin Shells, Delft, 1959

[3] S.R. Idelsohn, A family of conforming finite elements for the analysis of deep shell structures, Internat. J. Numer. Meths. Engrg., to appear.

[4] G. Dupuis, Application of Ritz' method to thin elastic shell analysis, J. Appl. Mech. 38 (1971) 987-996.

[5] G. Dupuis and J.J. Goel, A curved finite element for thin elastic shells, Internat. J. Solids and Structures 6 (1970).

[6] K. Marguerre, Zur Theorie der Cartrummten Platte Grosser Formänderung, Proc. 5th Internat. Congress of Applied Mech., pp. 93-101, summarized in: The Finite Trusting and Bending of Heated Elastic Lifting Surfaces (Bisplinghoff, R.L.).

[7] S.R. Idelsohn, Analyoes statique of dynamique Doctorat, Rapport L.T. (1974).

[8] A.C. Scordelis and K.S. Lo, Computer analysis of

[9] G. Bonnes, G. Dhatt, shells, 2nd Conf, on Matrix Methods in Structural 1 (e

[10] M.D. Olson, Analysis of arbitrary shells using shallow shell Research Serie Report, University of British Columbia (1971).

[11] G.E. Strickland and W. Loden, A doubly curved triangular shell element, 2nd Conf. on Matrix Methods in

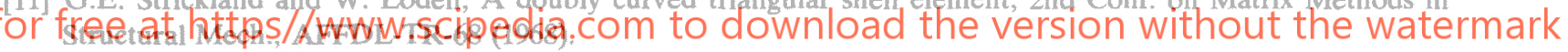

[12] S.R. Idelsohn, Un elément fini de coque triangulaire mince pour l'analyse statique des structures, Report SF-21, Lab, de Techn. Aéro. et Spatiales, Université de Liège (1972).

[13] M. Hogge, Famille d'eléments finis de coque plan cinématiquement admissibles pour l'analyse statique des structures, Rapport SF-22, Lab. de Tech. Aéro. et Spatiales, Université de Liège (1974).

[14] J.H. Argyris and N. Lochner, On the application of the SHEBA element, Comput. Meths. Appl. Mech. Engrg. 1(3) (1972).

[15] D.G. Ashwell, Strain elements, with applications to arches, rings and cylindrical shells, in: Finite Elements for Thin Shells and Curved Members (Wiley, London, 1976) Ch. 6.

[16] D.J. Dawe, Some high-order elements for arches and shells, in: Finite Elements for Thin Shells and Curved Members (Wiley, London, 1976) Ch. 8.

[17] G.R. Thomas and R.H. Gallagher, A triangular element based on generalized potential energy concepts, in: Finite Elements for Thin Shells and Curved Members (Wiley, London, 1976) Ch. 9.

[18] H. Parisch, A critical survey of the 9-node degenerated shell element with special emphasis on thin shell application and reduced integration, Comput. Meths. Appl. Mech. Engrg. 20 (1979) 323-350.

[19] J.H. Argyris and P.C. Dunne, On the application of the natural mode technique to small strain large displacement problems, Main invited Lecture, World Congress on FEM in Structural Mechanics, Bournemouth, U.K., Oct. 1975.

[20] E. Carnoy, Famille d'eléments hybrides déplacements du type délinquant pour lanalyse linéaire des structures, Rapport SF-84, L.T.A.S., Université de Liège (1978). 